\title{
DEFORMATIONS OF ISOLATED EVEN DOUBLE POINTS OF CORANK ONE
}

\author{
R. SMITH AND R. VARLEY
}

(Communicated by Ted Chinburg)

\begin{abstract}
We give a local deformation theoretic proof of Farkas' conjecture, first proved by Grushevsky and Salvati Manni, that a complex principally polarized abelian variety (ppav) of dimension 4 whose theta divisor has an isolated double point of rank 3 at a point of order two is a Jacobian of a smooth curve of genus 4. The basis of this proof is Beauville's result that a 4 dimensional ppav is a non-hyperelliptic Jacobian if and only if some symmetric translate of the theta divisor has singular locus which either consists of precisely two distinct conjugate singularities $\{ \pm x\}$ or has an isolated singular point which is a limit of two distinct conjugate singularities. We establish an explicit local normal form for the theta function near an isolated double point of rank 3 at a point of order two, which implies the point is such a limit (after translation to the origin), i.e. has a small deformation within the family defined by the universal theta function whose nearby singularities include two conjugate ordinary double points (odp's). The existence of such a deformation depends only on the facts that the theta function is even, a general theta divisor is smooth, and a general singular theta divisor has only odp's, also proved by Beauville in dimension 4 . The argument yields a similar result, also proved by Grushevsky and Salvati Manni, for ppav's of dimension $g>4$ whose theta divisor has an isolated double point of rank $(g-1)$, i.e. corank one, at a point of order two.
\end{abstract}

\section{INTRODUCTION}

Hershel Farkas conjectured [F, pp. 37-38] in 2004 the following statement designed to complete the geometric Schottky problem in genus 4: if the theta divisor on a 4 dimensional complex principally polarized abelian variety (ppav) $(A, \Theta)$ has a double point of rank 3 at a point of order two for the group law, then $(A, \Theta)$ is a Jacobian of a smooth curve of genus 4 (which then has a vanishing even theta null), or at least a product of Jacobians. This conjecture was proved by Grushevsky and Salvati Manni in 2006 in G-SM1 and completes the Andreotti-Mayer (and classical) program of characterizing Jacobians of genus 4 curves among all 4 dimensional ppav's by the singular points on $\Theta$.

Recall that in the moduli space $\mathcal{A}_{g}$ of $g$ dimensional principally polarized abelian varieties, Andreotti and Mayer $\left[\mathrm{A}-\mathrm{M}\right.$ ] focused on the "discriminant locus" $\mathcal{N}_{0}$ of those ppav's $(A, \Theta)$ for which $\Theta$ is singular, as well as "higher discriminant loci" where $\Theta$ is highly singular. More precisely, if $\mathcal{J}_{g}$ is the locus of Jacobians and products of Jacobians in $\mathcal{A}_{g}$, and if $\mathcal{N}_{d}=\left\{(A, \Theta) \in \mathcal{A}_{g} \mid \operatorname{dim}(\operatorname{Sing}(\Theta)) \geq d\right\}$, they

Received by the editors December 31, 2008 and, in revised form, April 6, 2009; July 3, 2010; and May 24, 2011.

2010 Mathematics Subject Classification. Primary 14-xx; Secondary 32-xx. 
showed when $g \geq 4$ that $\mathcal{J}_{g}$ is an irreducible component of $\mathcal{N}_{g-4}$. In particular in genus $g=4, \mathcal{J}_{4}$ is an irreducible component of the ordinary discriminant locus $\mathcal{N}_{0}$. Beauville $\left[\mathrm{B}\right.$ ] then showed that for $g=4$, the only other component of $\mathcal{N}_{0}$ is $\theta_{\text {null }}$, by definition the locus of ppav's $(A, \Theta)$, such that (a symmetric translate of) $\Theta$ has a point of positive even multiplicity at a point of order 2 for the group law of $A$, i.e. a "vanishing even theta null". Thus if $g=4, \mathcal{N}_{0}=\mathcal{J}_{4} \cup \theta_{\text {null }}$.

The proof by Grushevsky and Salvati Manni of Farkas' conjecture completes Andreotti and Mayer's and Beauville's work and enables one to read from $\operatorname{Sing}(\Theta)$ exactly which ppav's $(A, \Theta)$ belong to $\mathcal{J}_{4}$ and which to $\theta_{\text {null }}$. Their proof involves a detailed analysis of relations satisfied by theta functions and their derivatives using the heat equation. One corollary of the present argument is that, in addition to the standard fact that the theta function is even, the only information needed for a proof of Farkas' conjecture is already available for genus 4 theta functions in Beauville's thesis [B] in 1977: namely, $\mathcal{N}_{0}=\mathcal{J}_{4} \cup \theta_{\text {null }}$, a general theta divisor is smooth, and for a general ppav in $\theta_{\text {null }}$ the only singularity of the theta divisor is one ordinary double point (odp; see definition below).

It was known classically that the theta divisor of a non-hyperelliptic genus 4 curve whose canonical model lies on a rank 3 quadric in $\mathbb{P}^{3}$ has a corresponding isolated rank 3 double point at a point of order two in the Jacobian, hence a vanishing theta null, and represents a ppav in the intersection of the two components $\mathcal{J}_{4}, \theta_{\text {null }}$ of $\mathcal{N}_{0}$. If $\Theta$ has a non-isolated singularity it follows from [B, p. 181, Prop. (6.6)] that $(A, \Theta)$ is either a hyperelliptic Jacobian or a product of Jacobians, so to prove Farkas' conjecture it suffices to show that a ppav with an isolated rank 3 double point at a point of order two on its theta divisor must lie on $\mathcal{J}_{4}$. Our argument uses deformation theory of such a theta divisor to show that the ppav lies on at least one local analytic component $Z$ of $\mathcal{N}_{0}$ at whose general point the singular locus of $\Theta$ either has more than one singularity or has a double point which is not ordinary. Since by [B] the local component $Z$ cannot be in $\theta_{\text {null }}$, where generically there is just one singularity and that singularity is ordinary, it must lie in $\mathcal{J}_{4}$. Thus in $\mathcal{A}_{4}$, a symmetric theta divisor with an isolated rank 3 double point at a point of order 2 is a limit of theta divisors with conjugate odp's, i.e. is a limit of Jacobian theta divisors, hence a theta divisor either of a Jacobian or a product of Jacobians. Since the theta divisor on a product has no isolated singularities, it is in fact the theta divisor of a Jacobian.

Recall that the rank of a double point is the rank of its quadric tangent cone. A double point on a hypersurface in $\mathbb{C}^{n}$ is ordinary (an odp) if that rank is $n$, i.e. maximal, while a double point of rank $n-1$ is said to have "corank one". Our approach, if abstracted, shows that in any family of even analytic hypersurfaces, in which the general hypersurface is smooth and the general singular hypersurface has only ordinary double points, an isolated double point of corank one at the origin is always a limit of a pair of conjugate odp's on nearby hypersurfaces. We prove only the deformation theoretic statements and rely on Beauville for known facts about theta divisors in $\mathcal{A}_{4}$. For an application to isolated even double points of corank one on higher dimensional theta divisors, we appeal to the analogous facts proved by Debarre [D1] and by Grushevsky and Salvati Manni G-SM2.

The singularity theory of an isolated even double point of corank one provides a model for the structure of local analytic components of the discriminant locus $\mathcal{N}_{0}$ that pertain to the given singular point and for the way in which such components 
intersect. Then with the help of a simple consequence of the heat equation for the theta function, it is easy to see that there is a smooth local analytic component of $\theta_{\text {null }}$ that is tangent to a local analytic component of $\mathcal{N}_{0}$ not contained in $\theta_{\text {null }}$. Such a phenomenon was noted for $g=4$ by Rauch [Ra], extended to $g=5$ by [S-V2] and all higher $g$ by Debarre [D1, and then extensively analyzed by Grushevsky and Salvati Manni G-SM2. We discuss this tangential intersection result in the last section.

Theorem 1 (Characterization of genus 4 Jacobians). Let $(A, \Theta)$ be a 4 dimensional complex ppav, where $\Theta$ is a symmetric theta divisor on A. Also, let $\operatorname{dim} \operatorname{Sing}(\Theta)$ denote the dimension of the locus of singular points on $\Theta$. Then:

i) $A$ is a product of lower dimensional Jacobians iff $\operatorname{dim} \operatorname{Sing}(\Theta)=2$.

ii) $A$ is a hyperelliptic Jacobian iff $\operatorname{dim} \operatorname{Sing}(\Theta)=1$.

iii) $A$ is a non-hyperelliptic Jacobian with no vanishing even theta null iff $\Theta$ has exactly 2 singularities at "conjugate" points, i.e. points of the form $x$ and $-x$.

iv) $A$ is a non-hyperelliptic Jacobian with a vanishing even theta null iff $\Theta$ has an isolated rank 3 (corank one) double point at a point of order two.

The "only if" statements are classical. The "if" statements in parts i), ii), iii) are due to Beauville, and in iv) to Grushevsky and Salvati Manni. See [B] Thm., p. 149; (6.6), p. 181; (7.4), p. 184; (7.5), p. 191] and [G-SM1.

In this paper we prove a local structure theorem which implies the following proposition for genus 4 theta functions. Let $\mathcal{H}_{4}$ denote the Siegel space of symmetric $4 \times 4$ complex matrices with positive definite imaginary part, and consider an even theta function $\vartheta(x ; s)$ on $\mathbb{C}^{4} \times \mathcal{H}_{4}$. Thus for fixed $s_{0} \in \mathcal{H}_{4}, \vartheta\left(x ; s_{0}\right)$ is an even function of $x$ in $\mathbb{C}^{4}$ and $\vartheta\left(x ; s_{0}\right)=0$ defines the theta divisor over $s_{0}$ in $\mathbb{C}^{4}$. The standard theta functions with characteristics can be found in [F-K] $\S$ VI.1, pp. 302304]. The quotients of $\mathbb{C}^{4}$ and of $\vartheta\left(x ; s_{0}\right)=0$ by the lattice in $\mathbb{C}^{4}$ generated by the columns of the $4 \times 8$ normalized period matrix associated with a point $s_{0}$ in $\mathcal{H}_{4}$ realize a ppav $(A, \Theta)$ in $\mathcal{A}_{4}$. The coordinates $s$ on $\mathcal{H}_{4}$ will be centered with $s_{0}=0$.

Proposition 2. Let $\vartheta(x ; s)$ denote the universal theta function on $\mathbb{C}^{4} \times \mathcal{H}_{4}$, where $x=\left(x_{1}, x_{2}, x_{3}, x_{4}\right)$, and assume that $(x ; s)=(0 ; 0)$ is an isolated rank 3 double point of the fiber $\{\vartheta(x ; 0)=0\}$ in $\mathbb{C}^{4} \times\{0\}$ over $s=0$ in $\mathcal{H}_{4}$. Then there is an analytic family of equivariant local coordinate changes $(x ; s) \rightarrow(z ; s)$, which transforms $\vartheta(x ; s)$ to (a unit times) a sum of 3 squares plus the standard even deformation of $z^{4}$. That is, locally near $(0 ; 0)$, there exist analytic functions $z(x ; s)$ with $z(-x ; s)=-z(x ; s)$ such that $(z ; s)$ are coordinates in which $\vartheta(z ; s)$ equals a unit times a polynomial of the form

$$
z_{1}^{2}+z_{2}^{2}+z_{3}^{2}+z_{4}^{4}+b(s) z_{4}^{2}+c(s) .
$$

Here $b, c$ are analytic functions on Siegel space $\mathcal{H}_{4}$ near $s=0$ such that $b(0)=$ $c(0)=0$. Hence both divisors $\{c(s)=0\}$ and $\left\{b(s)^{2}-4 c(s)=0\right\}$ are contained in $\mathcal{N}_{0}$. Furthermore, all components of $\{c(s)=0\}$ are contained in $\theta_{\text {null }}$, but no component of $\left\{b(s)^{2}-4 c(s)=0\right\}$ is contained in $\theta_{\text {null }}$.

This implies Farkas' conjecture as follows.

Corollary 3. A four dimensional ppav $(A, \Theta)$ whose theta divisor has an isolated double point of rank 3 at a point of order two is a Jacobian of a smooth nonhyperelliptic curve of genus 4 with a vanishing even theta null. 
Proof of Corollary 3. First we translate $\Theta$ in $A$ so that the given isolated double point of rank 3 is at the origin, and then we use the analytic description provided by the proposition. Since $b(0)=c(0)=0$, the point $(A, \Theta)$ corresponding to $s=0$ lies on some component $Z$ of the divisor $\left\{b(s)^{2}-4 c(s)=0\right\}$, and by the proposition $Z$ is contained in $\mathcal{N}_{0}$ but not in $\theta_{\text {null }}$. Since by $[\mathrm{B}$ the only component of the discriminant locus $\mathcal{N}_{0}$ in $\mathcal{A}_{4}$ other than $\theta_{\text {null }}$ is $\mathcal{J}_{4},(A, \Theta)$ lies on $\mathcal{J}_{4}$ but is neither a product of Jacobians nor a hyperelliptic Jacobian, since by A-M] or again from [B], on those ppav's $\Theta$ has no isolated singularities. The double point of order two then represents the vanishing even theta null on the corresponding smooth non-hyperelliptic curve.

Remark. The logical structure of the proof below is to deduce Farkas' conjecture first from a less precise even Weierstrass polynomial representative of $\vartheta(x ; s)$ of degree $2 k \geq 4$ in $x_{4}$. After we know $(A, \Theta)$ is a Jacobian, we compute that the degree in the variable $x_{4}$ is equal to 4 .

\section{MOTIVATION FROM DEFORMATION THEORY OF SINGULARITIES}

Although the argument does not appeal to general theorems of deformation theory, it is suggested by them. One knows that every family of local hypersurface singularities which specializes to a given isolated singularity is pulled back by a classifying map from one standard model family determined by that singularity. Hence the structure of the model family plus the structure of the classifying map determines the local structure of any family specializing to that singularity. In particular, the equation of an isolated double point of "corank one" on a local analytic hypersurface in $\mathbb{C}^{n+1}$ can be expressed, after an analytic change of the independent variables, as (a unit times) a function of form $x_{n}^{2}+\ldots+x_{1}^{2}+x_{0}^{m}$, for $m>2$. The model family for this singularity is the space of monic polynomials of degree $m$ in $x_{0}$, without the $x_{0}^{m-1}$ term [A-G-V pp. 187-188, 150-151]. If the functions in the original family are also even, the equation can be expressed as $x_{n}^{2}+\ldots+x_{1}^{2}+x_{0}^{2 k}$, for some $k \geq 2$, and we prove in Lemma 4 below that after appropriate coordinate change and division by a unit, the family is defined by a function of the form

$$
x_{n}^{2}+\ldots+x_{1}^{2}+x_{0}^{2 k}+a_{k-1}(s) x_{0}^{2(k-1)}+\ldots+a_{1}(s) x_{0}^{2}+a_{0}(s),
$$

where the coefficients $a_{j}(s)$ define the classifying map and all $a_{j}(0)=0$. A reference for hypersurface deformations in the analytic case is $\mathrm{K}-\mathrm{S}$, and a reference for deformations of even hypersurfaces in the formal case is [Ri].

Thus for a theta function with an isolated double point of corank one at a point of order 2 , the model is that of a generic even polynomial $y^{2 k}+a_{k-1}(s) y^{2(k-1)}+$ $\ldots+a_{1}(s) y^{2}+a_{0}(s)$, specializing to the monomial $y^{2 k}$ for $s=0$. The discriminant locus of the polynomial $y^{2 k}+a_{k-1}(s) y^{2(k-1)}+\ldots+a_{1}(s) y^{2}+a_{0}(s)$, i.e. those values of $s$ where this polynomial has a multiple root in $y$, consists of two components: one is the locus $a_{0}(s)=0$, where generically there is one odp at $y=0$; the other is the discriminant locus $\{\delta(a(s))=0\}$ of the polynomial $t^{k}+a_{k-1}(s) t^{k-1}+\ldots+$ $a_{1}(s) t+a_{0}(s)$. I.e. if $h(t)=t^{k}+a_{k-1}(s) t^{k-1}+\ldots+a_{1}(s) t+a_{0}(s)$ is a polynomial of degree $k$ with a multiple root at $t$, putting $t=y^{2}$ gives an even polynomial $h\left(y^{2}\right)=y^{2 k}+a_{k-1}(s) y^{2(k-1)}+\ldots+a_{1}(s) y^{2}+a_{0}(s)$ of degree $2 k$ with multiple roots at both square roots of $t$. A generic such polynomial $h(t)$ has a single odp at some $t \neq 0$, and $h\left(y^{2}\right)$ has 2 "conjugate" odp's at $y=\sqrt{t}$ and $y=-\sqrt{t}$. 
In some sense this explains why $\mathcal{N}_{0}$ has two components and the structure of generic singularities of theta on those components. In particular, near a ppav with an isolated double point of corank one at $x=0$ on $\Theta$, the family of all ppav's is locally the pullback of the general "even" deformation of the singularity $y^{2 k}$ by a classifying map whose image contains generic points of both components of the discriminant locus.

\section{Proof of Proposition 2}

Step one (Reduction of the analytic equation to a polynomial). We give a local normal form or "model" for a family of arbitrary even analytic functions specializing to one with an isolated double point of corank one at the origin by using the Weierstrass preparation theorem iteratively to produce a polynomial generator for the ideal generated by the germ of the function. Recall from the language of group actions that a function is "equivariant" if it commutes with the group action and "invariant" if it ignores the action. Thus an invariant function is one that is constant on orbits, while an equivariant function only takes orbits to orbits. For example, with respect to the minus map, an even function is invariant, while an odd function is equivariant. Since we want to change coordinates while preserving the meaningfulness of the concept of evenness of the theta function in $x$, we want to use only coordinate changes which are equivariant for the minus map in the variable $x$ or are "odd" in $x$. In general we will use "iota" $(\iota)$ to denote the involution taking $(x ; s)$ to $(-x ; s)$.

Lemma 4 (Preparation Lemma). Assume:

- $U$ is an open neighborhood of $(0 ; 0)$ in $\mathbb{C}^{n+1} \times \mathbb{C}^{r}$ invariant under $\iota(x ; s)=$ $(-x ; s)$, where $r, n \geq 0$.

- $F(x ; s)$ is a complex-analytic function on $U$ invariant under $\iota$, i.e. $F(-x ; s)=$ $F(x ; s)$ (call such a function "even in $x ")$.

- $F(x ; 0)$ has an isolated corank one (i.e. rank $n)$ double point at 0 in $\mathbb{C}^{n+1}$ (where if $n=0$, this means that $F\left(x_{0} ; 0\right)$ is not identically zero but has no terms of degree two or less in $x_{0}$ ).

Then there exist an $\iota$-invariant open subset $U_{1}$ in $U$ containing $(0 ; 0)$ and complex analytic coordinates $z=z(x ; s)$ together with $s$ for $U_{1}$ such that $z(x ; s)$ is odd in $x$ (i.e. $z(-x ; s)=-z(x ; s)$ ) and in which $F$ has the following form:

- $F(x(z ; s) ; s)=u(z ; s)\left(z_{n}^{2}+\ldots+z_{1}^{2}+g\left(z_{0} ; s\right)\right)$, where

- $u(z ; s)$ is an analytic unit which is even in $z$, and

- $g\left(z_{0} ; s\right)=z_{0}^{2 k}+\sum_{0 \leq j<k} a_{j}(s) z_{0}^{2 j}$ is a monic even polynomial of degree $2 k$ in $z_{0}$ for some $k \geq 2$, and for all $j<k, a_{j}(s)$ is analytic and $a_{j}(0)=0$.

Proof of the Preparation Lemma. The proof will proceed by induction on $n \geq 0$. We start with the case $n=0$. Thus we have $F\left(x_{0} ; s\right)$ (in the single variable $\left.x=x_{0}\right)$, where $F$ is even in $x_{0}$. By hypothesis $F\left(x_{0} ; 0\right)$ vanishes at $x_{0}=0$ to some finite even order $2 k \geq 4$. Thus by the Weierstrass preparation theorem [G-R, p. 68], there is a unique polynomial in $x_{0}$ of the form $\left(x_{0}^{2 k}+\alpha_{2 k-1}(s) x_{0}^{2 k-1}+\ldots+\alpha_{0}(s)\right)$, with $k \geq 2$, where all coefficients $\alpha_{j}(s)$ are analytic in $s$ and all $\alpha_{j}(0)=0$, such that $F\left(x_{0} ; s\right)=u\left(x_{0} ; s\right)\left(x_{0}^{2 k}+\alpha_{2 k-1}(s) x_{0}^{2 k-1}+\ldots+\alpha_{0}(s)\right)$ on some neighborhood of $\left(x_{0} ; s\right)=(0 ; 0)$, and $u$ is an analytic unit. In particular, the unit 
is also unique. Thus since $F\left(x_{0} ; s\right)=F\left(-x_{0} ; s\right)$, it follows also that $F\left(x_{0} ; s\right)=$ $u\left(-x_{0} ; s\right)\left(\left(-x_{0}\right)^{2 k}+\alpha_{2 k-1}(s)\left(-x_{0}\right)^{2 k-1}+\ldots+\alpha_{0}(s)\right)$ is a representation of the same kind obtained by substituting $-x_{0}$ for $x_{0}$ in both the unit and the Weierstrass polynomial representing $F$. By uniqueness of the unit and the polynomial, both of them are even in $x_{0}$, and thus the polynomial has only even degree terms. Thus we get $F\left(x_{0} ; s\right)=u\left(x_{0} ; s\right)\left(x_{0}^{2 k}+\sum_{0 \leq j<k} \alpha_{2 j}(s) x_{0}^{2 j}\right)$, where $u$ is even in $x_{0}$. Then passing to a smaller $\iota$ invariant neighborhood $U_{1}$ of $\left(x_{0} ; s\right)=(0 ; 0)$ if necessary, and setting $z_{0}=x_{0}, u\left(z_{0} ; s\right)=u\left(x_{0} ; s\right)$, and $g\left(z_{0} ; s\right)=\left(x_{0}^{2 k}+\sum_{0 \leq j<k} a_{j}(s) x_{0}^{2 j}\right)$ where $a_{j}(s)=\alpha_{2 j}(s)$, we have the desired form for $F$ when $n=0$. (In this case no change of the coordinate $x_{0}$ was necessary.)

Now we carry out the inductive step. It will be necessary to have two "distinguished" indices for the variables in the course of the argument. Since the final result is that the exponents of all but one of variables are 2's, the remaining variable with higher exponent is distinguished in that sense. But in the induction, one of the variables whose exponent is 2 will be peeled off to carry out the induction, so that variable is also distinguished in a different sense. We will choose the variable $x_{0}$ (or $z_{0}$ ) as the one ultimately with exponent higher than 2 and choose $x_{n}$ as the variable to start the induction.

Fix $n>0$, consider $F(x ; s)=F\left(x_{0}, \ldots, x_{n} ; s\right)$ with an isolated rank $n$ double point in $x$ at $s=0$, and assume the stated structural result holds in the rank $n-1$ case, i.e. corank one on $\mathbb{C}^{n} \times \mathbb{C}^{r}$. After a homogeneous linear, hence $\iota$-equivariant, change of the coordinates $x=\left(x_{0}, \ldots, x_{n}\right)$, we may assume that for $s=0$ the quadratic term in $x$, which has rank $n>0$, has the form $x_{n}^{2}+$ a rank $(n-1)$ quadratic form in the variables $x_{n-1}, \ldots, x_{0}$.

Next by the Weierstrass preparation theorem, since $F\left(0, \ldots, 0, x_{n} ; 0\right)$ has order 2 in the variable $x_{n}$ (on a possibly smaller open neighborhood of $(0 ; 0)$ in $(x ; s)$ space, which may be arranged to be $\iota$-invariant),

$$
F(x ; s)=u_{1}(x ; s)\left(x_{n}^{2}+\alpha_{1}\left(x^{\prime} ; s\right) x_{n}+\alpha_{0}\left(x^{\prime} ; s\right)\right),
$$

where $u_{1}(x ; s)$ is a unit, $x^{\prime}$ denotes the coordinates $x^{\prime}=\left(x_{0}, \ldots, x_{n-1}\right)$ and the coefficient functions $\alpha_{1}\left(x^{\prime} ; s\right), \alpha_{0}\left(x^{\prime} ; s\right)$ in the quadratic Weierstrass polynomial are analytic and vanish at $\left(x^{\prime} ; s\right)=(0 ; 0)$. By the evenness of $F$ in $x$, the uniqueness of the unit and the polynomial, both of them are even in $x$, thus $u_{1}(-x ; s)=u_{1}(x ; s)$, $\alpha_{1}\left(-x^{\prime} ; s\right)=-\alpha_{1}\left(x^{\prime} ; s\right)$ and $\alpha_{0}\left(-x^{\prime} ; s\right)=\alpha_{0}\left(x^{\prime} ; s\right)$.

Now set $y_{n}=x_{n}-\left[\alpha_{1}\left(x^{\prime} ; s\right) / 2\right]$ and keep the other coordinates: $y_{j}=x_{j}$ for $j=n-1, \ldots, 0$. After this coordinate change we get

$$
F(x(y ; s) ; s)=u_{1}(x(y ; s) ; s)\left(y_{n}^{2}+\alpha_{0}\left(x^{\prime}\left(y^{\prime} ; s\right) ; s\right)\right)
$$

on a possibly smaller $\iota$ invariant neighborhood of $(0 ; 0)$ in $(y ; s)$ space. Moreover, $y(x ; s)$ is odd in $x$, so $x(y ; s)$ is odd in $y$, and both $u_{1}$ and $\alpha_{0}$ are even in $y$.

Now we want to apply the inductive hypothesis to the function $F^{\prime}\left(y^{\prime} ; s\right)=$ $\alpha_{0}\left(x^{\prime}\left(y^{\prime} ; s\right) ; s\right)$ which is analytic in $\left(y^{\prime} ; s\right)$ near $(0 ; 0)$ and even in the variables $y^{\prime}=\left(y_{0}, \ldots, y_{n-1}\right)$. As we have seen, $F^{\prime}$ is invariant for the involution $\iota^{\prime}:\left(y^{\prime} ; s\right) \rightarrow$ $\left(-y^{\prime} ; s\right)$, and $F^{\prime}\left(y^{\prime} ; 0\right)$ has a rank $n-1$ double point at $y^{\prime}=0$. Moreover, this singularity of $F^{\prime}\left(y^{\prime} ; 0\right)$ is isolated since $F(x(y ; 0) ; 0)$ has a singularity at $y=\left(y^{\prime}, 0\right)$ whenever $F^{\prime}\left(y^{\prime} ; 0\right)$ has a singularity at $y^{\prime}$, and the singular point of $F(x(y ; 0) ; 0)$ at $y=0$ is isolated. 
Thus by the inductive hypothesis there exists an $\iota^{\prime}$-invariant neighborhood $U^{\prime}$ of $\left(y^{\prime} ; s\right)=(0 ; 0)$, and complex analytic coordinates $\left(z^{\prime} ; s\right)$ on $U^{\prime}$ centered at $(0 ; 0)$, where $z^{\prime}\left(y^{\prime} ; s\right)$ is odd in $y^{\prime}$, and

$$
F^{\prime}\left(y^{\prime}\left(z^{\prime} ; s\right) ; s\right)=v\left(z^{\prime} ; s\right)\left(z_{n-1}^{2}+\ldots+z_{1}^{2}+g\left(z_{0} ; s\right)\right) \text {. }
$$

Here $v$ is a unit which is even in $z^{\prime}$, i.e. $v\left(-z^{\prime} ; s\right)=v\left(z^{\prime} ; s\right)$, and $g\left(z_{0} ; s\right)=z_{0}^{2 k}+$ $\sum_{0 \leq j \leq k-1} a_{j}(s) z_{0}^{2 j}$ is a polynomial of degree $2 k$ in $z_{0}$ for some $k \geq 2$, with only even degree terms, and analytic coefficients $a(s)$ such that all $a_{j}(0)=0$.

Now substitute this last form of $F^{\prime}\left(y^{\prime}\left(z^{\prime} ; s\right) ; s\right)$ for the $\alpha_{0}\left(x^{\prime}\left(y^{\prime} ; s\right) ; s\right)$ term above in the expression for $F(x(y ; s) ; s)$. We get

$$
F(x(y ; s) ; s)=u_{1}(x(y ; s) ; s)\left(y_{n}^{2}+v\left(z^{\prime} ; s\right)\left(z_{n-1}^{2}+\ldots+z_{1}^{2}+g\left(z_{0} ; s\right)\right)\right) .
$$

Extracting a complex analytic, hence even, square root of the even unit $v\left(z^{\prime} ; s\right)$, we introduce $z_{n}$ so that $z_{n}^{2}=y_{n}^{2} / v\left(z^{\prime} ; s\right)$. Then $z(y ; s)$ is odd with respect to the $y$ 's and $y(z ; s)$ is odd with respect to the $z$ 's. If we write $x(z ; s)$ for $x(y(z ; s) ; s)$ and $u(z ; s)=u_{1}(x(z ; s) ; s) v\left(z^{\prime} ; s\right)$, then $z(x ; s)$ is odd with respect to the $x$ 's and $x(z ; s)$ is odd with respect to the $z$ 's, and we finally get $F(x(z ; s) ; s)=u(z ; s)\left(z_{n}^{2}+z_{n-1}^{2}+\right.$ $\left.\ldots+z_{1}^{2}+g\left(z_{0} ; s\right)\right)$, where the unit $u$ is even in $z$ and where $g$ is as described in the preparation lemma.

By the preparation lemma, every deformation of an isolated even corank one double point at zero is modeled on deformations of the monomial $y^{2 k}$ within the space of even polynomials. Hence we next review the singularities of such polynomials.

Step two (The local polynomial model). Let $V=$ the space of monic even polynomials $f(y)=y^{2 k}+\sum_{0<j<k} a_{j} y^{2 j}$ of degree $2 k$ in the variable $y$, parametrized by $a=\left(a_{0}, \ldots, a_{k-1}\right)$ in $\mathbb{C}^{k} \cong V$ and where $k \geq 2$. This space is isomorphic to the space of monic polynomials $h(t)$ of degree $k$ by setting $t=y^{2}$. The discriminant locus in $V$ is the subset $D$ of those even monic polynomials $f(y)=h\left(y^{2}\right)$ having a repeated root. We keep this meaning of the notation $f$ and $h$ for the rest of the discussion. One can see that $D$ has two components, as follows.

Components $D_{0}$ and $D_{1}$ of the discriminant locus of the model. The repeated roots, or singular points, of a given even polynomial $f(y)$ are the common zeroes of $h\left(y^{2}\right)=0=\frac{d}{d y}\left(h\left(y^{2}\right)\right)=2 y \cdot h^{\prime}\left(y^{2}\right)$. Thus the singularities of the even polynomial $f(y)$ are precisely these: $y=0$ in case $h(t)$ has zero constant term, and $y= \pm \sqrt{t_{0}}$ in case $h(t)$ has a multiple root $t_{0}$. In particular, if $f$ has a singularity at a non-zero value of $y$, then it has more than one singularity. The discriminant locus $D$ is then the union of $D_{0}=$ those $f(y)$ where $h(t)$ has zero constant term and $D_{1}=$ those $f(y)$ where $h(t)$ has a repeated root. Moreover, the irreducibility of $D_{0} \cong \mathbb{C}^{k-1}$ is obvious, and that of $D_{1}$ is classical (cf. [S-V3]).

The critical locus of the model. Define the critical locus of the family of even polynomials $f(y)$ parametrized by $V \cong \mathbb{C}^{k}$ as the subset $\mathcal{C}$ in $\mathbb{C} \times \mathbb{C}^{k}$ consisting of pairs $(y ; a)$, where $y$ is a repeated root of the polynomial $f(y)$ with coefficient vector $a$. The projection $\mathbb{C} \times \mathbb{C}^{k} \rightarrow \mathbb{C}^{k}$ restricts to a map $\pi: \mathcal{C} \rightarrow D$ such that if $a$ is the coefficient vector of the polynomial $f(y)$, then $\pi^{-1}(a)$ has one point for each repeated root of $f(y)$.

At a general point $a$ of $D_{0}, \pi^{-1}(a)$ is the singleton $\{(0 ; a)\}$ corresponding to the unique singularity $y=0$ of the polynomial $f(y)$. At a general point $a$ of $D_{1}$, 
$\pi^{-1}(a)=\left\{\left(y_{0} ; a\right),\left(-y_{0} ; a\right)\right\}$, where $y_{0}^{2}=t_{0} \neq 0$ is the unique multiple root of $h(t)$. Thus $\pi$ has degree one over $D_{0}$ and degree two over $D_{1}$. If $a$ is in $D_{1}$ and $\pi^{-1}(a)$ is a singleton, then $h(t)$ has a singular point at $t=0$, hence $h(t)$ is divisible by $t^{2}$ and $f(y)$ is divisible by $y^{4}$. Hence the polynomial $X_{1}^{2}+\ldots+X_{g-1}^{2}+f\left(X_{g}\right)$ has a singularity at $X=0$ which is not an odp when $f$ is of this type.

By Lemma 4, the model for an isolated even singularity of corank one is the monomial $y^{2 k}$ with $k \geq 2$, which lies on $D_{0} \cap D_{1}$ and has a unique singularity at $y=0$. Since the map $\pi$ is proper, all polynomials near $y^{2 k}$ must have all their singularities near $y=0$. Thus by the discussion in the previous paragraph, if $f(y)$ is a polynomial near $y^{2 k}$ in $D_{1}$ and with only one singularity near $y=0$, then $X_{1}^{2}+\ldots+X_{g-1}^{2}+f\left(X_{g}\right)$ has a singularity at $X=0$ but not an odp. This remark will be used crucially in the next step.

Step three (Application to the case of theta functions). Next we deduce a general result for theta functions with an isolated double point of corank one at a point of order two. Recall $\mathcal{H}_{g}$ denotes Siegel space of genus $g$ and $\mathcal{A}_{g}=\mathcal{H}_{g} / \operatorname{Sp}(2 g, \mathbb{Z})$ the moduli variety of $g$ dimensional ppav's. We assume as known that the AndreottiMayer locus $\mathcal{N}_{0}$ parametrizing ppav's in $\mathcal{A}_{g}$ with singular theta divisor has exactly two irreducible components for $g \geq 4$, called $\mathcal{N}_{0}^{\prime}$ and $\theta_{\text {null }}$. Moreover, a general theta divisor over $\mathcal{N}_{0}$ has only odp's as singularities, two of them over $\mathcal{N}_{0}^{\prime}$ and one of them over $\theta_{\text {null }}$ [B], D1], G-SM2], S-V1], [S-V2]. Since we calculate with a theta function in the variables $(x ; s)$ on $\mathbb{C}^{g} \times \mathcal{H}_{g}$, by abuse of notation we will denote by $\mathcal{N}_{0}, \mathcal{N}_{0}^{\prime}$, and $\theta_{\text {null }}$ the inverse images in $\mathcal{H}_{g}$ of those same divisors in $\mathcal{A}_{g}$.

Theorem 5. Let $s=0$ be a point of $\mathcal{H}_{g}$ such that $g \geq 4$ and the corresponding theta function $\vartheta(x ; 0)$ defines an isolated double point of corank one at a point of order two for the group operation on the corresponding abelian variety. Then the point $s=0$ which lies on $\theta_{\text {null }}$ by hypothesis also lies on the other component $\mathcal{N}_{0}^{\prime}$ of the Andreotti-Mayer locus $\mathcal{N}_{0}$.

Proof. We may choose local coordinates $(x ; s)$ on $\mathbb{C}^{g} \times \mathcal{H}_{g}$, equivariant under $\iota$ so that the given point of order two is $(0 ; 0)$ and $\vartheta(x ; s)$ is even in $x$. Since $(x ; s)=$ $(0 ; 0)$ is an isolated double point of corank one, Lemma 4 above implies there is a neighborhood of $(0 ; 0)$ of form $\Delta_{1} \times \ldots \times \Delta_{g} \times W$ in $\mathbb{C}^{g} \times \mathcal{H}_{g}$, where the $\Delta_{j}$ are discs about 0 in $\mathbb{C}$, such that the restriction of $\vartheta(x ; s)$ to this neighborhood has in suitable coordinates, and up to a unit multiple, the form $X_{1}^{2}+\ldots+X_{g-1}^{2}+\left(X_{g}^{2 k}+\right.$ $\left.a_{k-1}(s) X_{g}^{2(k-1)}+\ldots+a_{1}(s) X_{g}^{2}+a_{0}(s)\right)$, for some $k \geq 2$, with all $a_{j}(s)$ holomorphic on $W$ and all $a_{j}(0)=0$. We regard the $a_{j}$ as defining a holomorphic map $a: W \rightarrow V$, where $V=$ the complex vector space of even polynomials $h\left(X_{g}^{2}\right)$ of degree $2 k$ over $\mathbb{C}$. Since $a(0)=(0, \ldots, 0)=\left(X_{g}\right)^{2 k}$, by shrinking $W$ we can arrange that for all $s$ in $W$, all roots, hence all singularities of $\left(X_{g}^{2 k}+a_{k-1}(s) X_{g}^{2(k-1)}+\ldots+a_{1}(s) X_{g}^{2}+a_{0}(s)\right)$, lie in $\Delta_{g}$.

Since $a(0)$ is the coefficient vector of $X_{g}^{2 k}$ and $k \geq 2$, it lies on both components $D_{1}$ and $D_{0}$ of the discriminant locus in $V$. We will argue that the pullback $a^{*}\left(D_{1}\right)=$ $D_{1}^{*}$ of the component $D_{1}$ is a divisor contained entirely in $\mathcal{N}_{0}$ but with no component contained in $\theta_{\text {null }}$, hence contained entirely in $\mathcal{N}_{0}^{\prime}$. In particular, when $g \geq 4, s=0$ lies on $\mathcal{N}_{0}^{\prime}$.

Since $y^{2 k}$ lies on both $D_{0}$ and $D_{1}$ in $V$ and a general theta divisor over $\mathcal{H}_{g}$ is smooth, the pullbacks $D_{0}^{*}$ and $D_{1}^{*}$ of $D_{0}, D_{1}$ by the map $s \mapsto a(s)$ are both non-empty (possibly reducible) divisors through 0 in $W \subset \mathcal{H}_{g}$. Since theta has 
singularities over all points of $D_{1}^{*}$ and $D_{0}^{*}$, both $D_{0}^{*}$ and $D_{1}^{*}$ are contained in the discriminant locus $\mathcal{N}_{0}$ of $\mathcal{H}_{g}$.

Now we are ready to argue that no component of $D_{1}^{*}$ lies in $\theta_{\text {null }}$. We see this as follows: since a component $Z$ of $D_{1}^{*}$ has the same dimension as $\mathcal{N}_{0}$, if $Z$ lies in $\theta_{\text {null }}$ it would contain a generic point of $\theta_{\text {null }}$; hence the singularity of $\Theta$ over a generic point of $Z$ would be a single odp, since that is the generic singularity over $\theta_{\text {null }}$. But over $D_{1}^{*}$ and near $s=0$, the critical locus of $\vartheta$ contains an isomorphic copy of the full critical locus of some polynomial $f(y)=y^{2 k}+\sum_{0 \leq j<k} a_{j} y^{2 j}=h\left(y^{2}\right)$ in $D_{1}$. If this were only one point, we remarked above that the corresponding critical point over $D_{1}$ would be at $y=0$ and of multiplicity $\geq 4$ for $f$, since $f(y)=h\left(y^{2}\right)$ and $h(t)$ is a polynomial with singularity at $t=0$. Hence the singularity of $\Theta$ would be that of the polynomial $\left(x_{1}^{2}+\ldots+x_{g-1}^{2}+f\left(x_{g}\right)\right)$, where $x_{g}^{4}$ divides $f\left(x_{g}\right)$. Then the singularity at $x=0$ would be a corank one double point, and not an odp, which contradicts the known structure of singularities over a general point of $\theta_{\text {null }}$. Thus every component $Z$ of $D_{1}^{*}$ is contained in $\mathcal{N}_{0}$ but not in $\theta_{\text {null }}$. Hence every such $Z$ lies in the other sublocus $\mathcal{N}_{0}^{\prime}$ of $\mathcal{N}_{0}$ in $\mathcal{H}_{g}$, where the generic singularity on $\Theta$ is two odp's. The proof of the theorem is now complete.

Remark. Since the map $\pi$ from critical to discriminant locus in the model family of polynomials is proper, the non-zero singularities $\{x,-x\}$ produced in the previous argument over a general point of $D_{1}^{*}$ converge to $(x ; s)=(0 ; 0)$, as $s \rightarrow 0$ in $\mathcal{H}_{g}$. Thus an isolated double point of corank one at a point of order two is a limit of the two ordinary double points on some nearby singular $\Theta$ divisors.

Step four (Computation of the Milnor number in genus 4). Now we can deduce Proposition 2. Everything has been argued in the proof of Theorem 5, except for the degree of the Weierstrass polynomial. So it remains only to compute $k$ for the polynomial $\left(x_{1}^{2}+x_{2}^{2}+x_{3}^{2}+x_{4}^{2 k}\right)$ which defines the genus 4 Jacobian theta divisor near the vanishing theta null. By the local algebra definition [A-G-V pp. 121, 242], the Milnor number of this isolated singularity equals $2 k-1$. We can compute this number globally topologically to be 3 as follows (cf. [S-V1]).

The sum of the Milnor numbers of all the singularities of $\Theta$ (the "global Milnor number") equals the difference between the Euler characteristic of $\Theta$ and the Euler characteristic of a generic smooth theta divisor. Hence the difference between the global Milnor numbers of the theta divisors of a generic Jacobian and a Jacobian with one vanishing even theta null equals the difference between their Euler characteristics.

For a genus 4 Jacobian $J(C)$ with one vanishing theta null, the theta divisor is the image by the Abel map of the symmetric cube $C^{(3)}$ of the curve $C$, which is an isomorphism except for collapsing one copy of $\mathbb{P}^{1}$ in $C^{(3)}$ onto the double point of $\Theta$, by the theorems of Abel and Riemann. Then by the long exact sequence for the "good pair" $\left(C^{(3)}, \mathbb{P}^{1}\right),[\mathrm{H}$, Thm. 2.13, p. 114], the Euler characteristic of $\Theta(C)$ is one less than that of $C^{(3)}$. For a generic genus 4 Jacobian $J(C)$, when the Abel map collapses two copies of $\mathbb{P}^{1}$, one onto each double point of $\Theta$, by making the same argument twice in succession, the Euler characteristic of $\Theta(C)$ is two less than that of $C^{(3)}$. The curves $C$ in these two calculations are analytically different, but they are homeomorphic, so their symmetric cubes $C^{(3)}$ are homeomorphic and have the same Euler characteristic. Thus the Euler characteristics of the two theta divisors are $\chi_{t o p}\left(C^{(3)}\right)-1$ and $\chi_{t o p}\left(C^{(3)}\right)-2$, and hence differ by one. 
Milnor showed that a singularity is an odp if and only if it has Milnor number 1. A generic Jacobian theta divisor has exactly two odp's as singularities and therefore has global Milnor number 2. Thus the global Milnor number of the theta divisor of a Jacobian with one vanishing even theta null is either 1 or 3 . Since the point is not an odp, it is 3 . That concludes the proof of Proposition 2.

\section{COMPARISONS AND GENERALIZATIONS}

The proof of Farkas' conjecture by Grushevsky and Salvati Manni in G-SM1. is a global one, establishing a containment relation between the closure of the set of genus 4 Jacobians with vanishing even theta null and the set of 4 dimensional ppav's with a double point of rank $<4$ at a point of order two, comparing the degree of these varieties in a projective embedding, and invoking Bezout's theorem to conclude equality as sets.

By [CM, Thm. 3] the only possible singularities of $\Theta$ on an indecomposable 4 dimensional ppav $A$ are double points. Since the argument in G-SM1 treats double points of all ranks less than 4 at points of order two, it follows that on a 4 dimensional ppav $A$ which is not in the closure of Jacobians but which has a singular theta divisor, not only do all the singularities occur at points of order two, but all of them are odp's. This describes all possible isolated singularities of $\Theta$ for 4 dimensional ppav's. Namely, if the theta divisor on a ppav $(A, \Theta)$ of dimension 4 has an isolated singularity at a point $p$ not of order two, then $p$ is a rank 4 double point and $(A, \Theta)$ is a non-hyperelliptic Jacobian. If there is an isolated singularity $p$ at a point of order two, then $p$ is a double point of rank 3 or 4 and $(A, \Theta)$ is a Jacobian if and only if the rank is 3 , in which case the Milnor number is also 3. By [V], D2 for ppav's in $\mathcal{A}_{4}$ not in the closure of Jacobians, the possible singularities of $\Theta$ consist of between one and ten isolated rank 4 double points at points of order two, and the maximum number of ten occurs on a unique 4 dimensional ppav.

It is argued in G-SM2 that if $g \geq 4$, the set of ppav's of dimension $g$ whose theta divisor has a point of order two with positive corank is equal to a specific locus contained in the part of the intersection of the two irreducible components of $\mathcal{N}_{0}$ along which the intersection scheme is nonreduced (cf. [D1, sect. 4]). Their argument uses the heat equation to show that the equations defining the two loci are the same. In a forthcoming work [S-V4] we strengthen the local arguments in this paper and deduce the results of [G-SM2, Prop. 8, hence Cor. 10] from general principles valid for even analytic hypersurfaces.

The local singularity theory also illuminates the structure of the intersection of the 2 global irreducible components $\theta_{\text {null }}$ and $\mathcal{N}_{0}^{\prime}$ of $\mathcal{N}_{0} \subset \mathcal{A}_{g}$ at a point $(A, \Theta)$ over which the (symmetric) theta divisor $\Theta \subset A$ has an isolated double point of corank one at a point of order 2 on $A$, assumed to be $(x ; s)=(0 ; 0)$. To get a picture of the local geometry, we should first look at the irreducible divisors $D_{0}$ and $D_{1}$ in our polynomial model $V$.

Elementary facts about the divisors $D_{0}$ and $D_{1}$ in $V$ and their intersection $D_{0} \cap D_{1}$. The divisor $D_{0}$ is smooth with linear equation $a_{0}=0$, and the divisor $D_{1}$ is irreducible with classical discriminant equation $\delta(a)=0$. In case $2 k=4$, the divisor $D_{1}$ is smooth; for $2 k \geq 6$, the divisor $D_{1}$ is analytically irreducible at 0 but is singular in codimension 1. It is known (cf. [S-V3]) that the tangent cone at 0 of $D_{1}$ is defined as a scheme by $a_{0}^{k-1}$. 
Now consider the structure of the intersection of $D_{0}$ and $D_{1}$. In case $2 k=4$, the intersection $D_{0} \cap D_{1}$ consists of a single irreducible component $R=\{(0,0)\}$ and the intersection scheme is non-reduced along $R$. Indeed, in the coordinates $b, c$ used previously on $V$ for the monic even polynomials $y^{4}+b y^{2}+c$ of degree $2 k=4$, $D_{0}$ has equation $c$ and $D_{1}$ has equation $b^{2}-4 c$, so the intersection scheme has ideal $\left(c, b^{2}\right)$. For $2 k \geq 6, D_{0} \cap D_{1}$ has 2 irreducible components $R$ and $S$ such that the intersection scheme is non-reduced along $R$ and is reduced generically along $S$. The points of $R$ correspond to those polynomials $y^{2 k}+a_{k-1} y^{2(k-1)}+\ldots+a_{2} y^{4}$ which are divisible by $y^{4}$, i.e. $R$ is defined by $a_{0}$ and $a_{1}$, and the intersection scheme of $D_{0}$ and $D_{1}$ has multiplicity 2 generically along $R$.

In the notation of step three, the intersection $D_{0}^{*} \cap D_{1}^{*}$ in $W$ is the pullback of the intersection $D_{0} \cap D_{1}$ in $V$. Let $R^{*}$ be the pullback of $R$. Notice that $D_{0}^{*}$ is defined by the function $a_{0}(s)$ on $W$ and $R^{*}$ is cut out on $D_{0}^{*}$ by the function $a_{1}(s)$ on $W$. Except for a unit factor, these functions are essentially the values of the theta function and its Hessian at $(0 ; s)$. Invoking the heat equation now allows a substantial simplification. It follows immediately that $D_{0}^{*}$ is smooth around $s_{0}$ in $W$ (cf. [S-V2]), since the theta divisor in $\mathbb{C}^{g}$ has a double point at $\left(0 ; s_{0}\right)$, and it is easy to see that the unit factor in the equation here does not cause a problem.

The tangential intersection property (local analytic version of some of the results of [D1, sect. 4], G-SM1, G-SM2]). For every local analytic component $Z$ of $D_{1}^{*}$, the smooth divisor $D_{0}^{*}$ meets $Z$ tangentially at all common points $s \in R^{*}$. Note that $s \in R^{*}$ iff the theta function $\vartheta(x ; s)$ on $\mathbb{C}^{g}$ still has a corank 1 double point at the origin $x=0$. Here, when we say that a smooth divisor meets a reduced, but possibly singular, divisor tangentially at a point $s$, we mean that the tangent space at $s$ to the smooth divisor is in the tangent cone at $s$ to the reduced divisor.

Proof. We have mentioned that by the heat equation, $D_{0}^{*}$ is smooth with scheme equation $a_{0}$ having a linear term $\ell$ (in the variables on $W$ around 0 ). On the other hand, since $a_{0}^{k-1}$ defines the tangent cone scheme at 0 to $D_{1}$ in $V$, the pullback scheme $D_{1}^{*}$ has tangent cone $\ell^{k-1}$ since there cannot possibly be any lower degree terms elsewhere in the pullback of the discriminant equation (thanks to the presence of $\ell$ in the pullback of $a_{0}$ ). Therefore any local analytic component $Z$ of $D_{1}^{*}$ must have a power of $\ell$ as its tangent cone equation, so $D_{0}^{*}$ meets $Z$ tangentially at 0 . Also, for any point $s \in D_{0}^{*} \cap Z$, if $s \in R^{*}$, then the intersection of $D_{0}^{*}$ and $Z$ remains tangential at $s$ by the same argument.

\section{ACKNOWLEDGEMENTS}

The authors wish to thank S. Grushevsky for useful correspondence and the referee for detailed help improving the exposition.

\section{REFERENCES}

[A-M] A. Andreotti and A. Mayer, On period relations for abelian integrals on algebraic curves, Ann. Scuola Norm. Sup. Pisa 21 (1967), 189-238. MR0220740 (36:3792)

[A-G-V] V. Arnol'd, S. Gusein-Zade, A. Varchenko, Singularities of Differentiable Maps. vol. I, Monographs in Mathematics, Birkhäuser, 1985. MR777682 (86f:58018)

[B] A. Beauville, Prym varieties and the Schottky problem, Invent. Math. 41 (1977), 149-196. MR.0572974 (58:27995) 
[CM] S. Casalaina-Martin, Cubic threefolds and abelian varieties of dimension five. II, Math. Z. 260 (2008), 115-125. MR2413346 (2009b:14090)

[D1] O. Debarre, Le lieu des variétés abéliennes dont le diviseur thêta est singulier a deux composantes, Ann. Sc. École Norm. Sup. 25 (1992), 687-708. MR,1198094(94a:14045)

[D2] O. Debarre, Annulation de thêtaconstantes sur les variétés abéliennes de dimension quatre, C.R. Acad. Sci. Paris Sér. I Math. 305 (1987), 885-888. MR925290 (89a:14055)

[F] H. Farkas, Vanishing theta nulls and Jacobians, in The Geometry of Riemann Surfaces and Abelian Varieties, Contemporary Mathematics, vol. 397, Amer. Math. Soc., 2006, 37-53. MR2217996 (2007c:14026)

[F-K] H. Farkas and I. Kra, Riemann Surfaces, 2nd ed., Springer-Verlag, 1992. MR.1139765 (93a:30047)

[G-SM1] S. Grushevsky and R. Salvati Manni, Jacobians with a vanishing theta null in genus 4, Israel J. Math. 164 (2008), 303-315. MR2391151 (2009e:14071)

[G-SM2] S. Grushevsky and R. Salvati Manni, Singularities of the theta divisor at points of order two, Int. Math. Res. Not. IMRN, 2007, no. 15, Art. ID, rnm 045, 15 pages. MR2348405 (2008g:14077)

[G-R] R. Gunning and H. Rossi, Analytic Functions of Several Complex Variables, PrenticeHall, 1965. MR0180696 (31:4927)

[H] A. Hatcher, Algebraic Topology, Cambridge University Press, 2002. MR 1867354 (2002k:55001)

[K-S] A. Kas and M. Schlessinger, On the versal deformation of a complex space with an isolated singularity, Math. Ann. 196 (1972), 23-29. MR0294701(45:3769)

[Ra] H. Rauch, The vanishing of a theta constant is a peculiar phenomenon, Bull. Amer. Math. Soc. 73 (1967), 339-342. MR0213544(35:4404)

[Ri] D. Rim, Equivariant G-structure on versal deformations, Trans. Amer. Math. Soc. 257 (1980), 217-226. MR.549162 (80k:14021)

[S-V1] R. Smith and R. Varley, On the geometry of $\mathcal{N}_{0}$, Rend. Sem. Mat. Univers. Politecn. Torino 42 (1984), 29-37. MR812628 (87c:14052)

[S-V2] R. Smith and R. Varley, Components of the locus of singular theta divisors of genus 5 , Algebraic Geometry, Sitges 1983, Springer Lecture Notes, 1124, Springer-Verlag, 1985, 338-416. MR805339 (86k:14030)

[S-V3] R. Smith and R. Varley, The tangent cone to the discriminant, Proceedings of the 1984 Vancouver Conference in Algebraic Geometry, Amer. Math. Soc., 1986, 443-460. MR.846034(88h:14019)

[S-V4] R. Smith and R. Varley, A splitting criterion for an isolated singularity at $x=0$ in a family of even hypersurfaces, Manuscripta Math. 137 (2012), 233-245.

[V] R. Varley, Weddle's surfaces, Humbert's Curves, and a certain 4-dimensional abelian variety, Amer. J. Math. 108 (1986), 931-952. MR853219 (87g:14050)

Department of Mathematics, University of Georgia, Athens, Georgia 30602

E-mail address: roy@math.uga.edu

Department of Mathematics, University of Georgia, Athens, Georgia 30602

E-mail address: rvarley@math.uga.edu 